\title{
Die Geschichte des/der Anderen: Zum Umgang mit dem österreichischen Täter-Opfer-Gedächtnis bei Maja Haderlap und Hamid Sadr
}

Die zunehmende Transkulturalisierung und Pluralisierung von Erinnerungskulturen provozieren sogenannte, clashes of memory' und Opferkonkurrenzen, und dies nicht nur auf transnationaler Ebene, sondern auch auf der Ebene des zwar national gedachten, aber immer heterogener werdenden kollektiven Gedächtnisses. In Österreich gibt es eine besondere Form des Umgangs mit Opfern, die im Gründungsmythos der Zweiten Republik, der das Land zum ersten Opfer des nationalsozialistischen Deutschlands erklärte, ihren Anfang genommen hat. Dieser Mythos ebnete nicht nur der Verklärung der jüngsten Vergangenheit den Weg, sondern auch dem Widerstreit konkurrierender partikularer Gedächtnisse, wobei Täter-Opfer-Positionen auf seltsame Weise miteinander vermischt bzw. vertauscht wurden (Botz 1997).

Am Beispiel dieses Umgangs mit den Opfern politischer und rassistischer Verfolgung lassen sich auch die wichtigsten Kritikpunkte der aktuellen Debatte um den Opferbegriff benennen: Opfernarrative können einerseits für kollektive Sinnstiftungen politisch missbraucht werden, indem die Binarisierung von Täterinnen und Opfern die Exklusion von Nicht-Zugehörigem erlaubt. Eine solche „Ethnisierung“ des Gedächtnisses und die nationale Fixierung der kollektiven Identität sind mit Assmann $(2013,147)$ in heterogenen und pluralen Gesellschaften besonders problematisch, zumal sie sowohl ethnisierten Minderheiten als auch Zugewanderten die Partizipation an der Aushandlung von identitätssichernden Erinnerungsnarrativen untersagen. Andererseits können durch Selbstviktimisierung Schuld und (Mit-)Täterschaft externalisiert werden, was wiederum - wie im Fall Österreichs - zur Verharmlosung von verübtem Verbrechen und zu Opferkonkurrenzen führt (Assmann 2013, 144-148).

Auch wenn die österreichische Literatur der 1950er und 1960er Jahre diese Opfererzählung teilweise bediente, haben kritische Texte, vor allem ab den 1980er Jahren maßgeblich zur Entlarvung der österreichischen ,Geschichtslüge“ beigetragen. Die inzwischen kanonisierten Werke und essayistischen Stellungnahmen - etwa Elfriede Jelineks Burgtheater (1985), Josef Haslingers Politik der Gefühle (1987) oder Thomas Bernhards Heldenplatz (1988) - spielen heute noch eine besondere Rolle in der gesellschaftlichen Auseinandersetzung mit dem historischen Erbe der NS-Vergangenheit. Als Teil der Erinnerungskultur haben diese 
Texte mit Astrid Erll (2011, 202) zum einen eine gedächtnisbildende Funktion, indem sie marginalisierte oder gar getilgte Erinnerungen in die Öffentlichkeit lancieren und auf diese Weise erzähl- und erinnerbar machen. Zum anderen können sie auch eine gedächtnisreflexive Funktion haben, indem sie die Erinnerungsvielfalt einer Gesamtgesellschaft präsentieren, Prozesse des Erinnerns und Vergessens inszenieren und reflektieren sowie Erinnerungshierarchien unterwandern. Beide Modi bewegen die Leser`innen zur Revidierung eigener Deutungsmuster, indem sie ihnen zur kritischen Beobachtung gesellschaftlicher Entwicklungen verhelfen (Neumann 2003, 67).

Auch die in diesem Beitrag behandelten Romane von Maja Haderlap und Hamid Sadr fungieren als Gedächtnismedien, die wichtige Diskurse der österreichischen Erinnerungskultur reflektieren. Der Roman Engel des Vergessens (2011) erzählt die aus dem kollektiven Gedächtnis der Österreicher^innen ausgeblendete Geschichte der Kärntner Slowen*innen und ihren Widerstand im Zweiten Weltkrieg. Haderlap wird damit einer speziell kärntner-slowenischen Erinnerung gerecht, gleichzeitig hinterfragt sie aber auch vorherrschende Sprechweisen der österreichischen Erinnerungspolitik und Geschichtsschreibung über den Kärntner Partisanenkampf (vgl. auch Spreicer 2015, 253). ${ }^{1}$ Ein ähnliches Ringen um eine andere Dimension des Gedächtnisortes des Zweiten Weltkriegs zeichnet Hamid Sadrs Der Gedächtnissekretär (2005) nach. Aus der Perspektive eines außenstehenden Fremden verfolgt er das Oszillieren zwischen Opfer- und Tätergedächtnis im österreichischen Kontext und greift das als längst überwunden geglaubte österreichische Opfernarrativ noch einmal kritisch auf. Der Roman liest sich jedoch nicht als simple Verurteilung der Tätergeneration, er fragt vielmehr nach Möglichkeiten alternativen Handelns und nach den Grenzen menschlicher Solidarität in einem transkulturellen Kontext, zumal sich hier jemand, der selbst Rassifizierungen und Ausgrenzung ausgesetzt ist (vgl. auch Mitterbauer 2011, 237), in die Position der Tätergesellschaft hineinversetzt, aber gleichzeitig den Opfern der NS-Verbrechen eingedenk bleibt.

Beiden Romanen ist gemeinsam, dass ihr^e Erzähler^in von den Rändern der Gesellschaft sprechen und die ethnisch fixierte Identitätserzählung der Österreicher^innen selbst in Frage stellen, indem sie „festgefügte Gedächtnisund Machtformationen“ (Assmann 2013, 144) zu verändern trachten. Dabei fragen sie auch danach, wie die Opfer in einem transkulturellen Kontext mit

1 An dieser Stelle möchte ich darauf hinweisen, dass unabhängig von meinem Beitrag zeitgleich ein Beitrag von Werner Wintersteiner (2019) zu Maja Haderlaps Roman Engel des Vergessens erschienen ist, der den Roman auf eine ähnliche Weise in der österreichischen Erinnerungspolitik verortet und behandelt. 
eigener Stimme sprechen können, ohne dass sie in den passiven Objektstatus im Sinne eines stellvertretenden ,Sprechens für und über jemanden‘ gedrängt werden.

Im Folgenden gehe ich anhand der beiden Texte der Frage nach, welche Rolle sie bei der narrativen Aushandlung des österreichischen Täter-OpferGedächtnisses spielen und welche literarischen Verfahren es ihnen erlauben, ihre gedächtnisbildende und -reflexive Funktion zu entfalten. Bevor ich auf die beiden Romane näher eingehe, soll jedoch jener komplexe erinnerungskulturelle Kontext beleuchtet werden, in dem die Werke verortet sind.

\section{Widersprüchlichkeiten österreichischer Erinnerungskulturen}

Die Disparität heutiger österreichischer Erinnerungskulturen lässt sich nicht nur von den spezifischen, vom Zweiten Weltkrieg herrührenden historisch-politischen Bedingungen herleiten, sondern auch von den gesamtgesellschaftlichen Entwicklungen eines immer schon mehrkulturellen und heterogenen Staates. Im österreichischen Kontext ist in diesem Sinne die Frage nach der Transkulturalität der Erinnerung im vielfachen Sinn von Bedeutung. Bereits Móritz Csáky (2011, 25-26) hat darauf hingewiesen, dass österreichische (wie auch zentraleuropäische) Erinnerungskulturen immer schon nur in ihrer immanenten Verflochtenheit wahrgenommen werden konnten. ,Kreolisierung‘ und Pluralität waren nicht nur für den Vielvölkerstaat der Habsburger Monarchie charakteristisch, sondern sie prägen weiterhin heutige Erinnerungskulturen der ehemaligen Kronländer auf der nationalen wie transnationalen Ebene (vgl. dazu Nagy 2019). Auf diese historischen Gegebenheiten zurückzuführen ist auch die zweite Dimension transkultureller Erinnerung in Österreich, nämlich das Nebeneinander des Gedächtnisses einer sich als Leitkultur verstehenden Mehrheitsgesellschaft und des ,Gegengedächtnisses‘ ethnisierter Minderheiten. In diese Erinnerungsvielfalt schreiben sich letztlich die partikularen Erinnerungen von Zugewanderten ein, die ihrerseits ebenfalls neue Narrative mit sich bringen und bestehende nationale Erzählmuster in Frage stellen. Diese Erinnerungen bilden die dritte Dimension transkultureller Erinnerung.

In den österreichischen Erinnerungskulturen wirken also verschiedene Erinnerungsgemeinschaften aufeinander und kämpfen stets um die Anerkennung ihrer jeweiligen identitätsstiftenden Erzählungen. Ich gehe hier insbesondere auf drei Konfliktlinien ein, die auch als Ergebnis von diversen transkulturellen Transformations- und Delegitimierungsprozessen zu lesen sind. 
Erstens ist hier die widersprüchliche Beurteilung des Endes des Zweiten Weltkriegs als Befreiung (in der Interpretation des Staates) oder als Niederlage zu nennen (im Sinne des deutschnationalen Bevölkerungsteils, vgl. dazu Rettl 2006, 12), mit der der sogenannte Opfermythos im engen Zusammenhang steht. ${ }^{2}$ Der mit der Unabhängigkeitserklärung von 1945 entstandene Gründungsmythos der Zweiten Republik ermöglichte nämlich nicht nur die Verleugnung von Schuld und Verantwortung, sondern auch die „Vielfachkodierung des Begriffs des Opfers“ (Lehnguth 2010, 119), ${ }^{3}$ die eine eigenartige „Täter-Opfer-Umkehr“ provozierte (Botz 1997, 232). Unmittelbar nach dem Krieg galten demnach nicht nur sämtliche zivile Opfer der Bombenangriffe als Kriegsopfer, sondern auch die Wehrmachtssoldaten, die ihr Leben - gemäß dem Heldenopfernarrativ - ,tapfer für das Land ,geopfert' hatten (Lehnguth 2010, 119). Auf diese Weise wurden tatsächliche Opfer der NS-Verfolgung bis in die 1980er Jahre aus dem österreichischen Gedächtnis getilgt. Erst die Erosion der Nachkriegsmythen in den 1980er Jahren sowie die Waldheim-Affäre 1986 haben in Österreich eine grundlegende Veränderung der Erinnerungskultur provoziert, die „mit der Neudefinition des Verhältnisses zur NS-Vergangenheit“ auch „ein Neuverhandeln der Geschichte“ (Uhl 2002, 221) mit sich brachte. Obwohl das Opfernarrativ allmählich von einem kritischen Tätergedächtnis abgelöst wurde, sind alte Gedächtnisformationen partiell heute noch wirksam. Im österreichischen Selbstverständnis herrscht immer noch ein sogenanntes „Täter-Opfer-Gedächtnis“ (Botz 1997) vor, das sich zwar die eigene Kollaboration und Mitverantwortung eingesteht und an eine supranationale Holocaust-Erinnerung (Lehnguth 2010) anschließt, aber dennoch auf dem eigenen Opfersein beharrt.

Der veränderte Umgang mit der NS-Vergangenheit ermöglichte also keineswegs, alle Opfer des nationalsozialistischen Regimes gleichermaßen zu rehabilitieren. Vor allem Erinnerungen sogenannter ethnischer Minderheiten wurden lange Zeit aus der kollektiven Erzählung der Nation verbannt. Hier deutet sich die zweite Konfliktlinie an: der ambivalente Umgang mit dem Widerstandskampf der Kärntner Slowen*innen, deren Situation eine spezielle ist, zumal die Mehrheit der Bevölkerung die Partisan*innen wegen ihres Widerstands des Landesverrates

2 Der gesellschaftliche Umgang mit dem Erbe des Zweiten Weltkriegs ist jedoch nicht auf diese zwei Positionen reduzierbar. Siehe dazu zum Beispiel Lehnguths Untersuchung, der vier verschiedene Gedächtnisformationen ausmacht, die auch in einzelne Parteipolitiken hineinwirken (Lehnguth 2010).

3 Assmann spricht über die doppelte Semantik des Wortes ,Opfer‘, das einerseits das „Heldenopfer“, andererseits das „Leidopfer“ bezeichnet und somit unterschiedliche Perspektiven und Selbstinszenierungen erlaubt (2013, 145-146). In Bezug auf den spezifischen Täter/Opfer-Diskurs in Österreich siehe auch Botz 1997. 
und des Verbrechens bezichtigt und ihnen den Opferstatus aberkennt, obwohl den vergleichsweise wenigen Partisan`innen nicht zuletzt der Staatsvertrag zu verdanken ist. Die auch politisch erzeugte Denunzierung kärntner-slowenischer Widerstandskämpfer^innen als Verräter`innen und „Kameradenmörder“ dient der Instrumentalisierung einer ganzen Volksgruppe als Feindbild, um einerseits die eigene Mittäterschaft (das Böse im Eigenen) zu verdrängen, andererseits um eine positive Identifikation mit dem Widerstand $\mathrm{zu}$ verhindern (Amann 2013, 89). Wie die Untersuchung von Amann zeigt, wurden literarische Texte über die traumatischen Erlebnisse dieser Gemeinschaft bis zum Erscheinen des Romans von Haderlap in der Öffentlichkeit kaum rezipiert, so dass die von ihnen erlebte Gewalterfahrung nicht zu Gehör kommen konnte. Diese Gedächtnislücke schließt nun der Roman Engel des Vergessens, dem es gelang, eine radikale Veränderung öffentlicher Erinnerungsdiskurse zu erwirken (vgl. dazu Wintersteiner 2019).

Schließlich muss der Blick auf Migrationsprozesse gelenkt werden, die mit einer zusätzlichen Pluralisierung von Erinnerungsformen von einst monokulturell vorausgesetzten Erinnerungskulturen einhergehen. Hier manifestiert sich also die dritte Konfliktlinie österreichischer Erinnerungskulturen zwischen den dominanten Narrativen einer Mehrheitsgesellschaft und der Perspektive von Zugewanderten, die dank ihrer verfremdenden Wahrnehmung - wie Grabovszki bemerkt (2009, 290) - einen neuen Blick auf Österreich erlauben und zu einer Umstrukturierung bestehender Wissensordnungen und Deutungsmuster sowie zur Destabilisierung gesellschaftlicher Selbst- und Fremdkonzepte beitragen können.

Die Verbindung von Erinnerung und Migration berührt in unserem Kontext noch eine weitere Problematik, nämlich, inwieweit Immigrant`innen in eine nicht nur nationalisierte, sondern auch ,ethnisierte‘ Erinnerungskultur eingebunden werden können/sollen, die die Erinnerung an die NS-Zeit als eine spezifisch deutsche bzw. österreichische Angelegenheit definiert (Assmann 2013, 128-129). Der Roman von Sadr ist in dieser Hinsicht von besonderem Interesse, weil er sich nicht nur souverän in das ursprünglich stark national definierte kollektive Gedächtnis einschreibt, sondern durch das Aufzeigen von strukturellen Ähnlichkeiten zwischen Antisemitismus und Rassismus (vgl. dazu auch Mitterbauer 2011, 238) und deren Ausgrenzungsmechanismen auf ein zentrales Defizit westeuropäischer Gesellschaften aufmerksam macht, nämlich den Verlust der Grundsolidarität mit Mitmenschen (vgl. dazu auch Assmann 2013, 138-139). Bei der Öffnung und nicht zuletzt auch Demokratisierung von Erinnerungskulturen ist deshalb eine multidirektionale Erinnerung von zentraler Bedeutung, d.h. die Anerkennung der Wechselseitigkeit und der dialogischen Natur der Erinnerungen, wobei die scharfe Trennung von ,eigener' und ,fremder' Erinnerung aufgehoben ist. Erst wenn der öffentliche Raum - im Sinne Rothbergs - nicht länger als 
„Kampffeld“, sondern als ein „dehnbarer diskursiver Raum“ konzipiert wird, in dem Erinnerungsgemeinschaften miteinander in Dialog treten und Erinnerungsnarrative ständig neu verhandeln (Rothberg 2009, 5), können sich neue politische Identitäten und neue Formen der Solidarität jenseits politischer und kultureller, nationalisierter sowie ethnisierter Grenzziehungen herausbilden (Rothberg 2009, 11). Die Theorie der multidirektionalen Erinnerung erlaubt für diesen Beitrag nicht zuletzt, die Romane miteinander in Beziehung zu bringen, thematisieren sie doch beide die Artikulationsmöglichkeiten minorisierter Gruppen in einem transkulturellen Erinnerungsraum, indem sie im oben beschriebenen Sinne neue Solidaritätsbündnisse ermöglichen. ${ }^{4}$

\section{Erzählen als Grenzüberschreitung: Engel des Vergessens}

„Sie [unsere Familiengeschichten] stehen in nahezu keiner Verbindung zur Gegenwart. Zwischen der behaupteten und der tatsächlichen Geschichte Österreichs erstreckt sich ein Niemandsland, in dem man verloren gehen kann" ( $E V$, 185). So beschreibt die Ich-Erzählerin die erinnerungs- und soziopolitische Situation, in der die Figuren des Romans Engel des Vergessens ${ }^{5}$ sich zu bewegen gezwungen sind. Damit problematisiert der autobiografisch gefärbte Familienroman am Schicksal dreier Generationen jene politischen Machtkämpfe, die Kärntner Slowen`innen weder diesseits (also in Österreich) noch jenseits der Grenze (in Slowenien) erlauben, den Partisanenkampf als ein zugleich nationales wie transnationales Handeln im „europäischen Kampf [...] gegen den Faschismus“ $(E V, 221)$ zu etablieren.

In diesem Sinne kann ihre Situation als eine Existenz im doppelten Niemandsland beschrieben werden. Die „tragisch verzerrte“ Gestalt der Kärntner Partisan ${ }^{\star}$ innen $(E V, 185)$ repräsentiert in Österreich eine Vergangenheit, die

\footnotetext{
4 Ein solches Solidaritätsbündnis ist erkennbar etwa im Bereich des Literaturmarktes, wo die kärntner-slowenischen Verlage wie „Drava“ und „Hermagoras/Mohorjeva“, die dezidiert eine Brückenfunktion übernehmen, sich sehr früh für die Literatur der zugewanderten Autor`innen engagierten. Das dritte Kapitel des Romans Der Gedächtnissekretär erschien zum Beispiel in der von Helmut A. Niederle herausgegebenen Anthologie Die Fremde in mir: Lyrik und Prosa der österreichischen Volksgruppen und Zuwanderer bei Hermagoras und Mohorjeva. Vgl. zum Verhältnis der Literatur der ethnischen Minderheiten und der Zugewanderten auch Sievers und Vlasta 2018.
}

5 Im Folgenden abgekürzt zitiert mit der Sigle $E V$, Seitenangabe. 
nicht nur marginalisiert und der offiziellen Geschichte des Landes gegenläufig ist, sondern auch eine unbequeme Geschichte darstellt, die ins „Kellerabteil“ eines zur Schau gestellten, prachtvollen „Hauses Österreich“ verbannt wird (EV, 186), um dort endgültig vergessen $\mathrm{zu}$ werden. Im transnationalen Kontext wiederum, „aus der slowenischen, zentralen Machtperspektive betrachtet“, wirken die Kärntner Partisan*innen ,wie Findlinge, die man aus der Revolutionsgeschichte fallen ließ“ $(E V, 223)$. So findet ihre Geschichte weder an nationale noch an transnationale Erinnerungskontexte Anbindung.

Dabei ist die Geschichte der Familie der Erzählerin unzertrennbar mit der ,großen' europäischen Geschichte, dem historischen Narrativ, verbunden: Großvater und Vater waren Partisanen, die Großmutter hat das Konzentrationslager Ravensbrück überlebt, drei aus der Wehrmacht desertierte Brüder der Mutter starben im Kampf auf der Seite der Partisan^innen. Die Geschichte der Familie ist somit - ähnlich wie andere kärntner-slowenische Familiengeschichten - zutiefst gezeichnet von Verlust, Gewalt und Tod, wobei Kriegsereignisse und erlebte Traumata transgenerationell in Gesprächen, etwa bei der Verrichtung der Arbeit, bei familiären Zusammentreffen, beim Feiern oder Trauern, mündlich weitergegeben werden. ${ }^{6}$ So erfährt die junge Erzählerin von Dachau und Mauthausen, von den Regeln des KZ, vom Verbrechen im Peršmanhof in Bad Eisenkappel und von den Foltern, die ihr Vater erlitten hat. Später, als junge Erwachsene, verfolgt sie in Wien die Machtmechanismen öffentlicher Erinnerungsdiskurse, die marginalisierte Erinnerungsnarrative konsequent aus dem offiziellen Gedächtnis ausschließen. Somit entscheidet sie sich, aus den zu Bruchstücken zerfallenen Geschichten eine zusammenhängende Erzählung zu formen (vgl. auch Banoun 2014, 22) - d.h. ihnen eine literarische Form zu geben, die es erlaubt, den generationellen Rahmen des kommunikativen Gedächtnisses zu verlassen und die Erzählungen in das kulturelle Gedächtnis zu heben.

Das Schreiben hat also zunächst eine politische Dimension, indem Haderlap wichtige öffentliche Diskurse der 1980er Jahre aufgreift, Fragen der Zugehörigkeit thematisiert und identitätspolitische Mechanismen der Grenzziehungen kritisch reflektiert.

Der Grenze wegen, die in den Augen der Mehrheit in unserem Lande nur eine nationale und sprachliche Grenze sein kann, muss ich mich erklären und ausweisen. Wer ich bin, zu wem ich gehöre, warum ich Slowenisch schreibe oder Deutsch spreche? Solche Bekenntnisse haben einen Schattenhof, in dem Gespenster herumstehen mit den Namen Treue und

6 Zur Funktion der oralen Erzähltradition in der Kärntner Erinnerungsgemeinschaft und des kommunikativen Gedächtnisses in Engel des Vergessens siehe auch Wagner 2013, 203-204; Banoun 2014, 21 und Amann 2013, 97. 
Verrat, Besitztum und Territorium, Mein und Dein. Das Überschreiten der Grenze ist hier kein natürlicher Vorgang, es ist ein politischer Akt. $(E V, 220)$

Die topografische Grenze geht dabei nicht nur in eine sprachliche über (Previšić 2014, 351), sondern auch in eine symbolische, die die Erinnerungsgemeinschaft der Kärntner Slowen`innen mitsamt ihren identitätsstiftenden Erzählungen von der vieler Österreicher^innen trennt und auf diese Weise unsichtbar macht.

Das Aufschreiben der Geschichte durch die Erzählerin hat also zweitens eine poetische Dimension, problematisiert doch der Roman die Erzählbarkeit der Geschichte des Partisanenkampfes und dessen Folgen angesichts der Fragmentiertheit, Sprunghaftigkeit und Unsagbarkeit der historischen Erfahrung des ${ }^{\star}$ der Einzelnen. Der Roman dokumentiert auf diese Weise nicht nur sein eigenes Entstehen, den Prozess der Auffindung der eigenen erzählerischen Stimme im „Stimmengewirr“ $(E V, 187)$, sondern reflektiert die sprachlichen Möglichkeiten der Artikulation traumatischer Erfahrungen, die Worte nie vollkommen präzise treffen können:

Ich kann nicht ergründen, was ich wirklich lebe. Meine Gefühle sind nicht mit den Wörtern vertraut, die ich spreche. [...] Früher nahmen die Empfindungen, so kam es mir vor, die Wörter an, jetzt aber bleibe ich mit allem zurück, wofür es keine Sprache gibt, und wenn es sie gibt, kann ich sie nicht in Dienst nehmen. $(E V, 100)$

Die Unverfügbarkeit und Unzulänglichkeit der Sprache zieht sich leitmotivisch durch den ganzen Roman und wird durch das hegemoniale Verhältnis des Deutschen und des Slowenischen weiter intensiviert (Čeh Steger 2014, 343; Previšić 2014, 350).

Das Schreiben hat auf diese Weise drittens eine zutiefst existenzielle Dimension, wird doch im doppelten Sinne eine verborgene, verdrängte Geschichte erzählt: die Geschichte von ,fremd‘ gemachten Anderen (nämlich der Kärntner Slowen`innen) und die Geschichte eines existentiellen Anderen im Eigenen, nämlich die des zerstörten, zerschundenen Körpers, der als Gedächtnisort und -medium Spuren der Gewaltgeschichte in sich trägt ${ }^{7}$ und eine sich in dieser Schmerzerfahrung manifestierende Erkenntnis offenbart (Wagner 2013, 201). So repräsentiert das Körpergedächtnis gefallener und gefolterter Partisan`innen

7 Zur Bedeutung des Körpergedächtnisses siehe auch Banoun (2014, 22). Für eine weitergehende Analyse sind m.E. die Beschreibung zerstörter oder leidender Körper $(E V, 110)$, das Abtasten des großmütterlichen Körpers nach Spuren des Grauens $(E V, 122)$, die Übernahme der Traumata, die sich in der Körpersprache manifestieren $(E V, 48-49)$, der Wunsch, aus dem Körper zu entfliehen $(E V, 96)$, oder sich einen neuen Körper zu ,erschreiben', von Relevanz. 
eine andersartige und sich anders artikulierende Wissensform, die nicht in die große Erzählung der Nation aufgenommen werden kann. Der Körper als Leidtragender markiert erneut eine Grenze zwischen Privatem und Politisch-Öffentlichem, die hier wiederholt auch entlang ethnisierter Kriterien abgesteckt wird. Dieser Körper, in den traumatische Erfahrungen eingeschrieben sind, verweist dabei nicht bloß auf Vergangenes, sondern auf eine Vergangenheit, die nicht vergeht und die eine Rückkehr in den alten Lebenszusammenhang unmöglich macht.

Kärntner Slowen*innen bleiben in der endlosen Wiederholung erlittener Gewalterfahrungen gefangen, in einer iterativen Zeit, die mit der linear ablaufenden historischen Zeit des Landes nicht in Übereinstimmung gebracht werden kann. Die Ich-Erzählerin kommuniziert zwischen diesen verschiedenen Zeitläufen, wobei sie ihre Position als ein zeitliches Niemandsland, eine Art ,Zeitkapsel beschreibt, in der Gegenwart und Vergangenheit immer gleichzeitig präsent sind:

Die Katrca habe einen wunden Rücken gehabt, sagt Großmutter und ich stelle mir, auf dem Bett liegend, den Rücken von Katrca vor, der in meiner Vorstellung aussieht wie ein bemaltes Tuch [...]. Hinter dem Rücken meiner Großmutter liegend, auf den erzählten Rücken von Katrca starrend, schwebe ich in der Vergangenheit wie in einem Zeittropfen, der in meinem Kopf kreist. $(E V, 126)$

Die Textstelle, die sich des Palimpsests als Gedächtnisfigur bedient (vgl. auch bei Banoun 2014, 21), um verschiedene Zeiten, Räume und Erinnerungssubjekte miteinander zu verbinden, verdeutlicht auch die Schreibstrategie, die den Erzählungen der Opfer gerecht werden will, indem sie ihnen das Sprechen aus der Subjektposition ermöglicht und jene leeren Stellen schreibend umkreist, die ihre toten Körper hinterließen. Es werden zahlreiche weitere Geschichten über Tote, Ermordete und Leidende aus dem Familien-, Verwandten- und Freundeskreis in die Erzählung eingewoben, die zwar mit fremder Stimme sprechen, aber schließlich als Teil der eigenen Geschichte erkannt werden und das Ich an einem gemeinsamen Narrativ teilhaben lassen. Ein zentraler Bezugspunkt dieses Narrativs ist das Lagerheft der Großmutter und die Briefe von ihren Mitgefangenen, die der Erzählerin helfen, die (Familien-)Geschichte zu rekonstruieren. ${ }^{8}$

8 Durch die Lagererfahrungen der Großmutter und ihre Briefe von Frauen aus dem KZ erhält der Text eine transnationale Dimension: „In Ravensbrück trafen die Frauen aus den Gräben mit den Frauen aus ganz Europa zusammen, vom Kärntner Rand in ein Zentrum des Krieges geschleppt, in dem sich die Lebenswege der Europäerinnen kreuzten.“ $(E V, 285)$ Dabei bleiben die konkreten Erzählungen dieser Frauen aus verschiedenen Ländern zwar eine Leerstelle (,was könnten sie erzählen, ausgehend von diesem Ort“), durch die Schicksalsgemeinschaft in diesem „Todesbrennpunkt“ $(E V, 285)$ werden ihre Gegenwart und ihre Vergangenheit für immer miteinander 
Nicht zufällig endet der Roman mit dem Bild der Großmutter, die in „trichterförmige[n] Baldachine[n]“ aus Wolle Stimmen einzufangen versucht $(E V, 287)$. Dieses Bild deutet einige zentrale poetologische Implikationen von Haderlap an. An ihm wird nicht nur die Literatur als aus vielen Erzählfäden gesponnene Textur sichtbar; es wird auch deutlich, dass das Gedächtnis selbst vielstimmig ist. ${ }^{9}$ Die scheinbar in der Luft freischwebenden Stimmen sind die Stimmen all jener, deren Leidensgeschichte der Roman in sich integriert, ohne sie zum stummen Erzählgegenstand zu machen. Die zentrale Setzung der Figur der Großmutter beschwört die ursprüngliche Situation des Geschichtenerzählens herauf und lässt dessen fundamentale Funktion für die Erinnerungs- und Identitätskonstruktion offensichtlich werden. Somit ist sowohl Erinnerung wie auch das damit transportierte Identitätsnarrativ ein vermitteltes und vielfach perspektiviertes. Die Geschichten sind aber auch auf eine Erzählinstanz angewiesen, die vom Standpunkt ihrer jeweiligen Gegenwart aus die disparaten Fäden einzusammeln, die darin artikulierenden Erzählstimmen wahrzunehmen und sie anzunehmen vermag. In diesem Sinne endet der Roman mit der folgenden Erkenntnis:

Der Engel des Vergessens dürfte vergessen haben, die Spuren der Vergangenheit aus meinem Gedächtnis zu tilgen. Er hat mich durch ein Meer geführt, in dem Überreste und Bruchstücke schwammen. Er hat meine Sätze auf dahintreibende Trümmer und Scherben prallen lassen, damit sie sich verletzen, damit sie sich schärfen. Er hat die Engelbildchen über meinem Kinderbett endgültig entfernt. Ich werde diesen Engel nicht zu Gesicht bekommen. Er wird keine Gestalt haben. Er wird in den Büchern verschwinden. Er wird eine Erzählung sein. (EV, 286-287)

Diese Poetologie kehrt die Benjamin'sche Denkfigur des Engels der Geschichte ins Aktive. ${ }^{10}$ Die vom Engel des Vergessens geführte Erzählerin kann nicht mehr tatenlos zuschauen, dem Fortschritt scheinbar ohnmächtig ausgesetzt. Sie muss die Bruchstücke zusammenfügen und in eine erzählbare und für alle sicht- und lesbare Geschichte verwandeln. Die Erzählung hat nicht nur das Ziel, eine kollektive Gewalterfahrung zu überwinden, indem die vielen Geschichten im Akt des Erzählens wiederholt und in einen größeren Erzählzusammenhang gebracht werden. Die vielen Geschlagenen, Gedemütigten und Ermordeten werden auch

verbunden. Diese gemeinsame Leiderfahrung ist auch die Grundlage einer transnationalen Erinnerung: „Ich will mir vorstellen, dass die Lagerfrauen mehr Verbindendes anführen könnten, als nationale Geschichtsschreibungen je zu formulieren und zu denken wagen.“ $(E V, 285)$

9 Čeh Steger (2014, 341) und Wagner (2013, 203-204) weisen darauf hin, dass die Erzählungen und Texte von Maja Haderlaps Verwandten wie die des Onkels Anton Haderlap, der Großtante Helena Kuchar und ihres Vaters Zdravko Eingang in den Romantext gefunden haben.

10 Vgl. die Analyse der Engel-Figuren im Roman bei Wagner 2013. 
explizit namentlich erwähnt $(E V, 141 ; E V, 240-245)$, um ihnen in diesem performativen Akt der Nennung ein Denkmal zu setzen und ihrem Handeln und Leiden Sinn zu verleihen.

In der Performativität der Übernahme des magischen Sprachdenkens der Großmutter, die die Erzählerin immer wieder auf die Macht des gesprochenen Wortes hinweist, ${ }^{11}$ und in der gleichzeitigen Rückkehr zur mütterlichen Tradition des geschriebenen (heiligen) Wortes kann die Erzählerin letztlich die anfängliche Unverfügbarkeit der Sprache überwinden und die scheinbar gegensätzlichen Positionen dieser beiden weiblichen Figuren miteinander vereinen. Die Erzählung, die letztlich auf dem Trümmer- und Scherbenhaufen der Geschichte in deutscher Sprache entsteht, dokumentiert den Prozess dieser Sprachfindung als Grenzüberschreitung, die mit dem Wechsel ins Deutsche nicht nur essentialisierende Bestimmungen von Identität und Zugehörigkeit durchkreuzt, ${ }^{12}$ sondern als politischer Akt ein gemeinsames Gedenken an die Opfer ermöglicht, das an alle Österreicher*innen adressiert ist.

\section{Doppelte Zeugenschaft: Der Gedächtnissekretär}

Der Roman Der Gedächtnissekretär ${ }^{13}$ des iranischen Autors Hamid Sadr erschien zum 50. Jubiläum des Staatsvertrags, gleichzeitig mit zwei weiteren Romanen, Es geht uns gut (Geiger 2005) und Vienna (Menasse 2005), die sich ebenfalls der Geschichte Österreichs zuwenden. Sadrs Text kann man aber nicht nur synchron im österreichischen Kontext verorten, sondern auch diachron in dessen kritischer literarischer Tradition, zumal er sich intertextuell auch auf Thomas Bernhards Heldenplatz bezieht, der wegen seiner scharfen Kritik an dem weiterwirkenden Antisemitismus und Faschismus im sogenannten Bedenkjahr 1988 für den bislang größten (Theater-)Skandal Österreichs sorgte. Im Vergleich zu den vorhin erwähnten Familienromanen von Geiger und Menasse war die Resonanz auf

11 In der Forschung wird immer wieder auf dieses magische Sprachdenken hingewiesen. Previšić bezeichnet das Kärntner Slowenische als „langue vernaculaire“ und „langue mythique“, wobei letztere sich vor allem in den Beziehungen weiblicher Figuren manifestiert (2014, 349). Mare betont die Nähe der Sprache der Großmutter zum „Zauberspruch“ (2015, 188). Ott (2017) spricht über die „Heiligkeit der Sprache“.

12 Auch Ott $(2017,108)$ interpretiert den Sprachwechsel mit Bezugnahme auf einige Selbstaussagen der Autorin als eine Möglichkeit, aus den nationalen und ethnischen Zuschreibungen auszubrechen.

13 Im Folgenden abgekürzt zitiert mit der Sigle GS, Seitenangabe. 
Sadrs Buch jedoch gering, wiewohl er eine besondere, „verschobene“ Perspektive auf Österreichs Vergangenheit (Vlasta 2016, 205) und das Täter-Opfer-Gedächtnis erlaubt.

Im Mittelpunkt des Romans steht Ardi, ein persischer Student, der, um Studium und Aufenthalt in Wien zu finanzieren, beim alten Herrn Sohalt, einem ehemaligen Nazi-Mitläufer, $\mathrm{zu}$ arbeiten beginnt. Seine Aufgabe ist es, mit Hilfe von Sohalts fünf „Oktavenheften“ Gebäude und Straßen von heute mit Fotografien aus dem Zweiten Weltkrieg zu vergleichen. Das Ziel der Dokumentationsarbeit des alten Herrn ist es, ein Buch mit ausgewählten Bildern über die zerbombte Stadt als „ein besonderes Andenken an Wien“ $(G S, 16)$ herauszugeben - ein Gedenkbuch also, das Sohalt ermöglicht, Wien zum Opfer der Kriegszerstörung zu stilisieren und dadurch seine eigene Täterschaft auszublenden. Unterschiedlicher könnten die Positionen der beiden Hauptfiguren nicht sein. Während Ardi von Anfang an am menschlichen Leid interessiert ist, registriert Herr Sohalts gleichgültiger Blick nur das Leblose zerstörter Gebäude und Straßen (vgl. auch Grabovszki 2009, 285; Vlasta 2016, 207). So legt sich auf das Bild über die unversehrte, vollkommen „renoviert[e] und saniert[e]“ $(G S, 18)$ Stadt Wien im Kopf von Ardi allmählich das Bild einer unerträglichen Totenstadt, um auf diese Weise ein Palimpsest zu bilden, in dem Vergangenheit und Gegenwart, Krieg und Frieden ununterscheidbar werden. Das seltsame ,Ineinanderrutschen“ der Zeiten geschieht plötzlich, ohne dass Ardi irgendeinen Einfluss darauf hätte (dazu vgl. Dabrowska 2012, 186-187):

Ich glaube, zwischen Schulhof und Tankstelle holte mich die Vergangenheit ein. Das Sichin-der-Zeit-glauben begann dort. Durch die staubige Luft wollte ich blind über die Straße laufen, als irgendwo ein offen gebliebener Fensterflügel zusammenschlug. [...] Vom Fliegeralarm gehetzt überquerten wir die Straße und rannten immer den weißen, in Leuchtfarbe gezeichneten Pfeilen nach, die uns dick auf die Hausmauern aufgetragen zum Luftschutzkeller führen sollten. $(G S, 53)$

Auf diese Weise wird die Hauptfigur in eine vergangene Realität eines anderen, ihm fremden Gedächtnisses derart hineingezogen, dass ihm seine eigene Identität, Sprache und Geschichte abhandenkommen (Grabovszki 2009, 258). Was als simple Arbeit als Kundschafter begann, wird für Ardi nach und nach zu einer existentiellen Aufgabe doppelter Zeugenschaft, die das Tätergedächtnis zu rekonstruieren und die aus ebendiesem Gedächtnis getilgten Erinnerungen an die (jüdischen) Opfer wieder sichtbar zu machen trachtet, selbst dann, wenn er selbst dabei zugrunde geht.

Das Aufdecken der Lügen und Fälschungen Herrn Sohalts, der durch die Unkenntlichmachung bestimmter Textstellen in seinen Oktavenheften und durch das Aussortieren nicht geeigneter Fotos die Spuren von Verbrechen ver- 
schwinden lässt, geschieht mit Hilfe von verschiedenen Erinnerungsträgern - Notizen, Briefen, Nazirequisiten und Familienfotos -, die es Ardi erlauben, eine verdrängte Geschichte wieder lesbar zu machen. Der Student rekonstruiert mitnichten nur die persönliche Nazi-Vergangenheit von Sohalt, ermöglicht doch das Aufeinanderkopieren der Zeitschichten, Orte und Ereignisse eine imaginierte Zeugenschaft, die über die grausamsten Verbrechen der Nationalsozialisten zu berichten weiß:

Ich marschierte mit ihm [Herrn Sohalt] von Wien nach Linz, von dort zum alten Steinbruch Wiener Graben, von dort zum Bettelberg, stieg dort aus, ging von Stein zu Stein, von Holzbaracke zu Holzbaracke, dabei kein gestreiftes Häftlingskleid, kein Gesicht und keinen Koffer außer Acht lassend, bis mir das Wort Sonderbehandlung geläufig war. (GS, 171)

Die Fotografien, die Sohalt einmal im KZ Mauthausen, ein anderes Mal an der Ostfront mit Wehrmachtssoldaten oder gerade bei der Enteignung jüdischen Eigentums zeigen, erlauben dem Studenten, den alten Herrn als NS-Funktionär zu identifizieren, der mitsamt seiner Familie tief in Kriegsverbrechen verwickelt war. Aber auch andere Bilder von gefolterten russischen Gefangenen sowie von Ermordeten und bei Bombenangriffen Umgekommenen in den Wiener Straßen werfen die Frage nach Sohalts tatsächlicher Rolle im NS-System auf. Diese Fotos bleiben insofern Leerstellen, als weder Ardi noch die LeserInnen erfahren, zu welchem Zweck und für wen Sohalt die Bilder gemacht hat und warum er sie besitzt. Da die Täter^innen schweigen, können die historischen Zusammenhänge nicht mehr vollständig rekonstruiert werden.

Ersichtlich werden die Diskrepanzen mehrheitsösterreichischer Erinnerung in der Wiener Stiftskirche, in der Ardi eines Tages die „Gedenktafel für die Gefallenen der Weltkriege“ entdeckt (GS, 91). Während darauf Soldaten, Majore und Generäle als heldenhafte Verteidiger der Heimat verehrt werden, sind die namenlosen Opfer der NS-Verbrechen aus öffentlichen Orten des Gedenkens getilgt.

Ihre Spuren kann Ardi lediglich in solchen Nicht-Orten der Erinnerung (nonlieux de mémoire ${ }^{14}$ ) wiederfinden, die zwar unauffällig, aber „unbestechlich“ (GS, 90) sind, und die mit kaum vernehmbarer Stimme eine andere Geschichte der Stadt flüstern. Die Pflastersteine und alten Wände Wiens, ${ }^{15}$ die voneinander weit

14 Der Begriff non-lieux de mémoire ist ein Wortspiel, das Pierre Nora’s Terminus des Gedächtnisortes (lieu de mémoire) aufgreift, jedoch im negativen Sinne. Es handelt sich um Erinnerungsorte, die aus dem Gedächtnis verschwunden sind bzw. die vergessen oder verdrängt wurden. Zur Verwendung des Begriffs vgl. David et al. 1999.

15 Vlasta (2016, 206-212) untersucht eingehend die Funktion dieses Gedächtnisses der Objekte (memory of things). 
entfernte Orte und Zeiten miteinander verbinden, fungieren auf diese Weise als Zeitzeugen:

Über die Steinbrüche Wiener Graben [in Mauthausen] und Bettelberg, die vor dem Krieg als Herstellungsort der Pflastersteine für Wien bekannt wurden, weiß ich nicht genug, es gab und gibt Gerüchte, flüsterleise Pflastergerüchte. (GS, 91)

Die Überblendung von Räumen und Zeiten signalisiert zugleich die Wiederkehr des Traumatischen in einem Differenzmoment: Ardi erlebt zwar im Akt des Hineinrutschens in die Vergangenheit die Bombardierungen mit und ist Augenzeuge von Folterungen und Ermordungen, die Ereignisse sind aber nur Produkte (s)eines Erinnerungs- und Vergegenwärtigungsprozesses, der in dieser Wiederholung die unauflösbare Diskrepanz von Damals und Heute und die Unwiederbringlichkeit der Toten offensichtlich werden lässt. Das Verwischen der Realitäten erreicht seinen Höhepunkt, als Ardi überlegt, wie er neun jüdische Männer, die sich im Keller des Hauses in der Förstergasse 2 verstecken, vor Denunziation und Ermordung retten könnte. Die Unmöglichkeit der Rückkehr in diese vergangene Welt verbietet ihm ein physisches Eingreifen. Erst die Umschreibung der Geschichte Sohalts ermöglicht ihm, an der Konstruktion und Dekonstruktion des kollektiven Gedächtnisses aktiv teilzuhaben.

In halluzinatorischen Zuständen rekonstruiert Ardi schließlich anhand von Sohalts Notizfragmenten und Bildern ${ }^{16}$ sowie entlang seiner eigenen phantasierten Erlebnisse die letzten Kriegstage und entscheidet sich, einen neuen, kohärenten Erinnerungstext zu schreiben, der die Täter`innen zwingt, ihre Vergangenheitsversion zu revidieren, „und zwar dort, wo sie wegen der Lächerlichkeit (oder Schändlichkeit) der Tatsachen in Gedächtnislücken verschwunden war“ (GS, 172). Daneben entsteht auch ein neuer Bildband, der Ardis Sicht auf die NS-Zeit wiedergibt, die zwar das Bild der Österreicher`innen zu korrigieren, aber keineswegs die Traumata zu überwinden vermag. Das „Nichtvergessenkönnen“ (Mitterbauer 2011, 239) treibt den Studenten schließlich in den Wahnsinn, so dass er sich in die psychiatrische Anstalt von Steinhof begibt. Hier beginnt er in Rückblenden die Geschichte seiner psychischen Erkrankung aufzuschreiben. Das ist der erzählerische Rahmen, in dem die Sohalt-Geschichte eingebettet ist und der die zeitliche Distanz zum Geschehen signalisiert. Eine Distanz, die das Niederschreiben der Geschichte erst erlaubt.

16 In diesem Sinne haben Sohalts Aufzeichnungen und Fotos eine ambivalente Funktion: Sie versuchen einerseits eine grausame Vergangenheit zu verdecken, andererseits aber ermöglichen sie Ardi, diese Vergangenheit überhaupt wahrzunehmen. 
Am Erzählort, der als literarischer Ort, als Gedächtnisort und als konkreter Ort des Schreibens gleichzeitig fungiert, werden gesellschaftliche und individuelle Prozesse des Erinnerns und Vergessens gleichsam reflektiert. Während Ardi das Otto-Wagner-Spital als einen „fotofreie[n], kriegszeitfreie[n] Ort“ (GS, 28) betrachtet, wo er vergessen kann, ist ausgerechnet dieser Ort alles andere als geschichtsneutral, steht doch der vom Autor ganz bewusst gewählte ehemalige Ort namens Spiegelgrund für die in der Nervenheilanstalt verübten NS-Verbrechen an Kindern und Jugendlichen (vgl. auch Mitterbauer 2011, 238). Als literarischer Ort verweist er u.a. auf Thomas Bernhard, auf dessen geisteskranke Figuren Sadr intertextuell Bezug nimmt, um die Ich-Dissoziation von Ardi und dessen Wahn als Reaktion auf die gesellschaftlichen Verhältnisse zurückzuführen. Dieses Leiden an den Menschen angesichts deren Zerstörungswut und Tötungslust ist ein Motiv, das auch Ingeborg Bachmann in ihrem Berlin-Essay Ein Ort für Zufälle aufgreift, in welchem die Krankheit ähnlich zum Roman Der Gedächtnissekretär ein ,Ineinanderrutschen' von Orten und Zeiten provoziert: In beiden Fällen „erzwingt“ - so Bachmann - die Beschädigung der Stadt „eine Einstellung auf Krankheit, auf eine Konsequenz von variablen Krankheitsbildern, die Krankheit hervorruft“ (Bachmann 1978, 279). Die Prosa von Bachmann und Sadr mag zwar halluzinatorisch erscheinen, vermag jedoch in dieser „Radikalisierung der Darstellung“ (Bachmann 1978, 279) einen exakten Befund über die Situation des Menschen in einer Gesellschaft zu vermitteln, die gerne jene Stimmen an die Ränder verbannt, die auf den immerwährenden Krieg in der jeweiligen Gegenwart aufmerksam zu machen wagen.

Der Spiegelgrund als Erzählort weist somit nicht nur auf die historische und gesellschaftliche Bedingtheit von Ardis Krankheit, sondern auch - ähnlich wie bei Haderlap - auf die Prozesse des Ausschlusses jegliches Fremden aus dem österreichischen Gedächtnis hin: auf die Exklusion des ,fremden' Ardi und mit ihm auf die Exklusion des Traumatischen als die eines unheimlichen Anderen, das außerhalb des Diskurses steht, von dem die Realität jedoch immer wieder heimgesucht wird. Zugleich ist der Ort ein individueller traumatischer Ort für den Erzähler, der, aus dem Sinnzusammenhang seiner Heimat und Familie herausgelöst und durch die Erlebnisse in Wien, eine radikale Entwurzelung erfährt. Auf diese Weise werden hier das Unheimliche der Gewalttaten und der Verlust der Heimat miteinander verschränkt - eine Verschränkung, die die existenzielle Heimatlosigkeit von Menschen im Exil offensichtlich werden lässt: „die Nähe von unheimlich und Heimweh macht mir da große Probleme. Wenn ich unheimlich sagen wollte, fiel mir sogleich der Flakturm in der Stiftkaserne ein. Beim Wort Heimweh standen die Bäume meiner Heimatstadt vor mir“ (GS, 29). Die Verbindung des Unheimlichen mit der Heimat verweist aber auch auf eine Erinnerung, die Ardi verdrängt, nämlich auf die Gewaltgeschichte seines Heimatlandes, die 
in seiner Erzählung vollkommen ausgeblendet wird. Auf diese Weise fungiert die österreichische NS-Vergangenheit mit Freud als eine „Deckerinnerung“, die die eigenen traumatischen Erinnerungen verdeckt. In diesem Kontext liest sich auch die Mahnung des iranischen Gedichts am Ende des Romans, das auf das Verkennen des Wesentlichen anspielt und ihn, Ardi, zum Handeln, nämlich zum Rückzug vom Trugbild der kriegerischen Vergangenheit eines anderen bewegt.

\section{Non-lieux de mémoire des Schreibens}

Die beiden Romane Engel des Vergessens und Der Gedächtnissekretär verbindet nicht nur, dass sie herrschende Diskurse über Täterschaft und Opfertum in der österreichischen Gesellschaft aus einer marginalen Position destabilisieren, sondern auch, dass sie dies mit ähnlichen literarischen Verfahren tun. Beide Romane veranschaulichen die Funktionsweise des kommunikativen Gedächtnisses, indem sie auf individuelle Erinnerungen und verschiedene Gedächtnisträger zurückgreifen und die Art und Weise der Überlieferung historischer Erfahrungen aufzeigen. Als „Protokoll[e] einer Spurensuche“ (Banoun 2014, 22) müssen sie Leerstellen füllen, das Nicht-Gesagte bzw. das Unsagbare verbalisieren. Während Sadrs Hauptfigur gefälschten Erinnerungen auf die Spur kommen und die Täter^innen zu einer kritischen Selbstreflexion zwingen will, ringt Haderlaps Roman mit der Artikulation traumatischer Erfahrungen. Somit problematisieren beide die Möglichkeiten des Erzählens angesichts der Unsagbarkeit, Unzuverlässlichkeit bzw. der Lückenhaftigkeit von Erinnerungen.

Sie besuchen dabei nicht nur geschichtsträchtige, symbolische Erinnerungsorte, sondern auch Nicht-Orte der Erinnerung, d.h. unscheinbare, auf den ersten Blick bedeutungsleere Orte, die die Spuren der Gewaltgeschichte in sich tragen. In Der Gedächtnissekretär sind es alte Möbelstücke, Wohnungen, Wände und Pflastersteine, die von vergangenen Verbrechen ein flüsterndes Zeugnis ablegen. In Engel des Vergessens erscheinen die Kärntner Landschaft, der Wald und die Bauernhöfe als Gedächtnisträger, in denen sich der Tod, die Vernichtung und die Zerstörung eingenistet haben und auf diese Weise die Erinnerung an die Opfer bewahren. ${ }^{\mathbf{1 7}}$ In beiden Romanen wird die Gegenwart von der Vergangenheit heimgesucht, die in beiden Fällen auch die Identität des bzw. der Schreibenden verunsichert und bedroht. So werden der^die Erzählerin auch körperlich

17 Zur Bedeutung der Landschaft als Gedächtnisträger und zur Zerstörung der Naturidylle vgl. Mare 2015, 198-203 und Čeh Steger 2014, 352. 
in die vergangene Geschichte anderer hineingezogen und wollen, zwischen Vergangenheit und Gegenwart pendelnd, etwas rekonstruieren, das sie persönlich nicht erlebt haben, das aber über den Umweg ihrer eigenen Erinnerungsarbeit integraler Teil ihres Selbst geworden ist. Um verschiedene Zeiten, Räume und Erinnerungssubjekte miteinander zu verknüpfen, greifen beide Texte den Palimpsest als Gedächtnis- und Gestaltungsfigur auf, der ihnen erlaubt, die Vielfachkodierung geschichtlicher Erfahrungen und die permanente Präsenz der Vergangenheit in der Gegenwart zu veranschaulichen.

Nicht zuletzt konstruieren sowohl Haderlap als auch Sadr mit der Vielstimmigkeit ihrer Erzählungen einen Erzählraum, der jenseits herkömmlicher Zugehörigkeitskoordinaten an die Gewaltgeschichte des Zweiten Weltkriegs und an die vielen individuellen Geschichten des Leids erinnert und gleichzeitig dem Gedächtnis der Opfer eingedenk bleibt - einem stets unbegreiflich bleibenden Anderen, das nur im Akt des Schreibens vom Niemandsland der Erinnerung zurückerobert werden kann.

\section{Literaturverzeichnis}

Amann, Klaus. „Kampfplatz Erinnerung: Der Widerstand der Kärntner Slowenen im Zweiten Weltkrieg als politischer und literarischer Topos“. Erfundene Erinnerung: Literatur als Gedächtnisbildung und Gedächtnisreflexion. Hg. Thomas Eder und Klaus Amann. Linz: StifterHaus, Zentrum für Literatur und Sprache, 2013. 81-107.

Assmann, Aleida. Das neue Unbehagen an der Erinnerungskultur: Eine Intervention. München: C. H. Beck, 2013.

Bachmann, Ingeborg: „Ein Ort für Zufälle“. Gesammelte Werke. Band 4. Hg. Christine Koschel, Inge von Weidenbaum und Clemens Münster. München und Zürich: Piper, 1978.

Banoun, Bernard. „Schmalspur-Bahn der Erinnerung: Aspekte des kommunikativen Gedächtnisses in Werner Koflers Tanzcafé Treblinka und Maja Haderlaps Engel des Vergessens“. Literatur - Politik - Kritik. Hg. Harald Jele und Elmar Lenhart. Göttingen: Wallstein, 2014. 17-24.

Botz, Gerhard. „Opfer/Täter-Diskurse: Zur Problematik des ,Opfer'-Begriffs“. Zeitgeschichte im Wandel. Hg. Gertraud Diendorfer. Innsbruck und Wien: StudienVerlag, 1997. 223-236.

Čeh Steger, Jožica. „Die zerstörte Dorfidylle an der österreichisch-slowenischen Grenze: Maja Haderlaps Engel des Vergessens“. Imaginäre Dörfer. Zur Wiederkehr des Dörflichen in Literatur, Film und Lebenswelt. Hg. Werner Nell und Marc Weiland. Bielefeld: transcript, 2014. 339-355.

Csáky, Móritz. „Kultur als Kommunikationsraum: Das Beispiel Zentraleuropas“. Gedächtnis und Erinnerung in Zentraleuropa: Jahrestagung des Literatur- und Kulturwissenschaftlichen Komitees der Österreichischen und Ungarischen Akademie der Wissenschaften. $\mathrm{Hg}$. András Balogh und Helga Mitterbauer. Wien: Praesens, 2011. 17-44.

Dabrowska, Anna. „Das gestohlene Trauma im Roman Der Gedächtnissekretär“. Traum und Trauma: Kulturelle Figuration in der österreichischen Literatur: Beiträge zur Jahrestagung 
der Franz Werfel-StipendiatInnen am 15. und 16. April 2011 in Wien. Hg. Arnulf Knafl. Wien: Praesens 2012. 182-191.

David, Thomas, Luc van Dongen und Marietta Meier. „Non-lieux de mémoire: Einleitung“. Traverse: Zeitschrift für Geschichte/Revue d'histoire 1 (1999): 7-11.

Erll, Astrid. Kollektives Gedächtnis und Erinnerungskulturen. 2. Auflage. Stuttgart und Weimar: Metzler, 2011.

Grabovszki, Ernst. „Österreich als literarischer Erfahrungsraum zugewanderter Autorinnen und Autoren“. Von der nationalen zur internationalen Literatur: Transkulturelle deutschsprachige Literatur und Kultur im Zeitalter globaler Migration. Hg. Helmut Schmitz. Amsterdam und New York: Rodopi, 2009. 275-292.

Haderlap, Maja. Engel des Vergessens. Göttingen: Wallstein, 2011.

Lehnguth, Cornelius. „Ende der Externalisierung? Die parteipolitische Auseinandersetzung um die NS-Vergangenheit in Österreich seit der Waldheim-Affäre“. Diktaturüberwindung in Europa: Neue nationale und transnationale Perspektiven. Hg. Birgit Hofmann. Heidelberg: Winter, 2010. 117-131.

Mare, Raffaella. „Ich bin Jugoslawe - ich zerfalle also“: Chronotopoi der Angst: Kriegstraumata in der deutschsprachigen Gegenwartsliteratur. Marburg: Tectum, 2015.

Mitterbauer, Helga. „Mnemosyne migriert: Gedächtnistransformationen in der österreichischen Gegenwartsliteratur“. Gedächtnis und Erinnerung in Zentraleuropa: Jahrestagung des Literatur- und Kulturwissenschaftlichen Komitees der Österreichischen und Ungarischen Akademie der Wissenschaften. Hg. András F. Balogh und Helga Mitterbauer. Wien: Praesens, 2011. 231-244.

Nagy, Hajnalka. „Vernetzte Geschichten? Nationalisierung und Transnationalisierung im mitteleuropäischen Erinnerungsraum“. Kontaminierte Landschaften: Mitteleuropa inmitten von Krieg und Totalitarismus: Eine exemplarische Bestandsaufnahme anhand von literarischen Texten. Hg. Alexander Höllwerth. Berlin u.a.: Peter Lang, 2019. 377-396.

Neumann, Birgit. „Literatur als Medium (der Inszenierung) kollektiver Erinnerungen und Identitäten“. Literatur - Erinnerung - Identität: Theoriekonzeptionen und Fallstudien. Hg. Astrid Erll, Marion Gymnich und Ansgar Nünning. Trier: WVT, 2003. 49-78.

Niederle, Helmut A. Hg. Die Fremde in mir. Lyrik und Prosa der österreichischen Volksgruppen und Zuwanderer. Ein Lesebuch. Klagenfurt/Celovec: Hermagoras/Mohorjeva, 1999.

Ott, Herta Luise. „Abschied von der ,Heiligkeit der Sprache‘: Maja Haderlaps Beitritt zur deutschsprachigen Literatur“. $L a(s)$ literatura(s) en lengua alemana y su apertura internacional/Deutsche Literatur(en) und ihre internationale Entgrenzung. Hg. María José Calvo und Bernd Marizzi. Madrid: Editorial DYKINSON, 2017. 105-118.

Previšić, Boris: „Polyphonien in der slowenisch-österreichischen Grenzzone Kärnten: Peter Handke, Maja Haderlap, Peter Waterhouse“. Philologie und Mehrsprachigkeit. Hg. Till Dembeck. Heidelberg: Winter, 2014. 341-358.

Rettl, Lisa. PartisanInnendenkmäler: Antifaschistische Erinnerungskultur in Kärnten. Innsbruck: StudienVerlag, 2006.

Rothberg, Michael. Multidirectional Memory: Remembering the Holocaust in the Age of Decolonization. Stanford: Stanford University Press, 2009.

Sadr, Hamid. Der Gedächtnissekretär. Wien: Deuticke, 2005.

Sievers, Wiebke, und Sandra Vlasta. „New Austria, Old Roots“. Immigrant and Ethnic Minority Writers since 1945. Hg. Wiebke Sievers und Sandra Vlasta. Leiden: Brill Rodopi, 2018. 43-76. 
Spreicer, Jelena. „Geschichte aus dem slowenischen Blickwinkel: Maja Haderlaps Engel des Vergessens". Narrative im (post)imperialen Kontext. Hg. Matthias Schmidt. Tübingen: Francke, 2015. 251-259.

Uhl, Heidemarie. „Konkurrierende Vergangenheiten: Offizielle Narrationen, ,Gegenerzählungen“ und Leerstellen des ,österreichischen Gedächtnisses“ in der Zweiten Republik“. Inszenierungen des kollektiven Gedächtnisses: Eigenbilder, Fremdbilder. Hg. Móritz Csáky und Klaus Zeyringer. Innsbruck: StudienVerlag, 2002. 220-235.

Vlasta, Sandra. Contemporary Migration Literature in German and English: A Comparative Study. Leiden und Boston: Brill Rodopi, 2016.

Wagner, Karl. „,Es wird eine Erzählung sein“: Maja Haderlaps Engel des Vergessens“. Krieg, Widerstand, Befreiung. Hg. Fabjan Hafner und Johann Strutz. Klagenfurt u.a.: Drava, 2013. 193-205.

Wintersteiner, Werner. „Angel of Oblivion: Literature and Memory Politics in Austria“. https:// www.tandfonline.com/doi/full/10.1080/13511610.2019.1578196?scroll=top\&needAcces $\mathrm{s}=$ true. Innovation: The European Journal of Social Science Research. London: Taylor and Francis Online, 2019 (1. März 2019). 
